\title{
VARIABILIDAD Y TENDENCIAS CLIMÁTICAS EN CHILE CENTRAL EN EL PERÍODO 1950-2010 MEDIANTE LA DETERMINACIÓN DE LOS TIPOS SINÓPTICOS DE JENKINSON Y COLLISON
}

\author{
Pablo Sarricolea Espinoza ${ }^{1,2}$ \\ Óliver Meseguer-Ruiz ${ }^{1}$ \\ Javier Martín-Vide ${ }^{1}$ \\ ${ }^{1}$ Grupo de Climatología. Universidad de Barcelona \\ ${ }^{2}$ Departamento de Geografia. Universidad de Chile \\ psarricolea@uchilefau.cl, oliver.meseguer@gmail.com,jmartinvide@ub.edu
}

\section{RESUMEN}

El propósito es conocer las situaciones sinópticas más características y sus tendencias, y cómo se asocian con las teleconexiones. La presencia del Anticiclón del Pacífico SurOriental es fundamental: los días anticiclónicos (ANT) alcanzan un 54\% de frecuencia, los tipos advectivos (ADV) (31\%) y los ciclónicos (CYC) (15\%). Las circulaciones del S y SE son frecuentes entre noviembre y marzo, mientras que las SW, W y NW destacan de mayo a septiembre. Se aprecia una disminución de los tipos anticiclónicos, siendo actualmente los advectivos los más frecuentes. El patrón de teleconexión mejor asociado a los tipos Jenkinson y Collison (J\&C) es la Oscilación Antártica, especialmente con el tipo anticiclónico y advectivo del sur.

Palabras clave: Chile, circulación atmosférica, Jenkinson y Collison, tendencias, teleconexiones.

\section{ABSTRACT}

The goal is to know the most characteristic weather types and their trends, and how they associate with teleconnection patterns. The presence of the Southeastern Pacific High is

Fecha de recepción: marzo 2012.

Fecha de aceptación: febrero 2013. 
essential: anticiclonic days (ANT) days catch up a frequency of 54\%, advective types (ADV) types a $31 \%$ and ciclonic days (CYC) days a $15 \%$. South and SE circulations are frequent between November and March, and SW, W and NW emphasize from May to September. High pressure types decrease, being actually the directional types the most frequent ones. The best associated pattern to the Jenkinson y Collison (J\&C) types is the Antarctic Oscillation, especially with the A and S ones.

Key words: Chile, atmospheric circulation, Jenkinson \& Collison, trends, teleconnections.

\section{INTRODUCCIÓN}

La Climatología sinóptica, entendida como el estado medio de los patrones de circulación atmosférica, se ha transformado en una eficaz y potente herramienta para la clasificación de los distintos tipos de tiempo, pues permite atribuir a dichos patrones unas determinadas situaciones meteorológicas y climáticas, por ejemplo, la ocurrencia de precipitaciones, de nevadas, de olas de calor y de frío, etc., entre otras.

Así, Yarnal et al. (2001) señala que uno de los aspectos fundamentales de la Climatología sinóptica corresponde al establecimiento de las relaciones empíricas entre la circulación atmosférica y el clima local.

Las primeras aproximaciones subjetivas para clasificar las circulaciones atmosféricas y los tipos de tiempo ocasionadas por ellas fueron llevadas a cabo, entre otros ${ }^{1}$, por Lamb (1972), quien a partir de los mapas de presión en superficie para las Islas Británicas, y desde 1861, distinguió las características de los tipos de tiempo ocasionados por los distintos flujos atmosféricos, destacando la variabilidad interanual y los ciclos de los períodos anticiclónicos, ciclónicos y advectivos de flujos del oeste y del este.

Entre los métodos tradicionales para establecer una climatología de los patrones de circulación atmosférica se encuentra la aplicación de una clasificación subjetiva a una serie de una o más décadas de mapas del tiempo. No obstante, al ser subjetiva, los resultados no son homogéneos, ni transferibles, pues dependen del punto de vista del observador. Aun así, hay ciertas reglas y situaciones modélicas de configuraciones sinópticas que el investigador puede aplicar con seguridad (Martín-Vide, 2005), pero el hecho de ser una clasificación manual implica un trabajo enorme para tratar la gran cantidad de información necesaria para cubrir el período analizado.

A partir de los métodos de clasificación sinóptica diaria es posible conocer cómo se distribuyen estadística y temporalmente los distintos tipos de circulación atmosférica. Lamb (1972) desarrolló una clasificación subjetiva que cuenta con 27 tipos (Lamb Weather Types, LWTs), la cual fue adaptada de modo objetivo y automático por El-Dessouky y Jenkinson (1975) para Egipto, utilizando 9 puntos de presión atmosférica reducida al nivel del mar,

1 Entre los primeros métodos subjetivos de clasificación encontramos a Hess \& Brezowsky (1952). También Lund (1963) clasifica patrones de circulación mediante correlaciones entre mapas del tiempo y configuraciones prototípicas. 
y luego aplicada por Jenkinson y Collison (1977) para las Islas Británicas, usando en total 16 puntos, siendo estos autores los que dan el nombre definitivo a esta clasificación sinóptica objetiva y automática. El método de Jenkinson y Collison (J\&C en adelante) es en la actualidad una de las clasificaciones objetivas más utilizadas, y ello se debe a la sencillez y eficacia que posee en la generación de series climáticas de tipos de tiempo. De hecho, la Climatic Research Unit (CRU) ha mantenido actualizada la clasificación diaria de J\&C hasta el presente, y la ha comparado con la clasificación de Lamb (Jones et al., 1993), obteniendo resultados muy similares, incluso con datos de reanálisis (Jones et al., 2013).

Existen numerosos trabajos que han utilizado el método de J\&C. Por citar algunos, en el norte de Europa los estudios de Linderson (2001), Stehlík (2001), Post et al. (2002) y Tang et al. (2009); en la Península Ibérica se ha aplicado por parte de Spellman (2000), Trigo y DaCamara (2000), Martín-Vide (2002), etc. El caso de la Península Ibérica es de particular interés para el caso de Chile central, pues representa a los climas de tipo mediterráneo, y, a partir de ahí, es posible comparar las tendencias de los tipos de circulación atmosférica. En este sentido, se observa para el Mediterráneo occidental un claro predominio de tipos indeterminados $(\mathrm{U})$ producto de situaciones de pantano barométrico $(27,2 \%$, sobre todo en verano), características de un ámbito cuasicerrado y cálido, seguido de situaciones anticiclónicas, las cuales representan el 20,7\% de los días entre 1948 y 2009 (Grimalt et al., 2013); asimismo, las tendencias indican que los días anticiclónicos decrecen, mientras que los días indeterminados y ciclónicos aumentan en las últimas décadas.

En el hemisferio sur y en Chile existen dos aplicaciones del método de J\&C. Una realizada por Frias (2008) y Frias et al. (2009), mediante dos mallas (una centrada en los $35^{\circ} \mathrm{S}$ y otra en los $55^{\circ} \mathrm{S}$ ) de 16 puntos cada una, y a partir de los datos de presión atmosférica del Proyecto ECWMF ERA-40 Reanalysis. Y la segunda, aplicada por Sarricolea et al. (2011) para Chile central utilizando 9 puntos y los datos del proyecto NCEP/NCAR Reanalysis, y para el período 1950-2010. Un antecedente significativo es que no se presenta ningún tipo indeterminado, y eso se debe a las condiciones de circulación y geográficas que rigen a Chile central, diferentes a las del Mediterráneo occidental.

Así, la circulación atmosférica que explica las características climáticas de Chile, y particularmente su zona central, está controlada por el anticiclón subtropical del Pacífico suroriental (APSO) y el cinturón de bajas presiones subpolares (CBPP). El APSO afecta a la zona comprendida entre los $30^{\circ}$ y $40^{\circ} \mathrm{S}$ de latitud, y el CBPP se localiza entre los $45^{\circ} \mathrm{S}$ y $55^{\circ} \mathrm{S}$ de latitud, desplazándose hacia latitudes más tropicales en invierno y favoreciendo el desarrollo de sistemas frontales que provocan parte de las precipitaciones de Chile central.

Cabe destacar que se desconocen las tendencias que han experimentado los distintos patrones de circulación atmosférica, y si ellas están moduladas por algún patrón de teleconexión. Por ello, nos planteamos verificar si los distintos tipos de J\&C son afectados por El Niño Oscilación del Sur (ENSO), mediante los índices de Oscilación del Sur (SOI) y el Índice Multivariado de El Niño (MEI); además de la Oscilación Decadal del Pacífico (PDO), la cual está relacionada con el ENSO (Zhang et al., 1997), y también, si los tipos de J\&C obtenidos para Chile central están relacionadas con la Oscilación Antártica (AAO).

$\mathrm{Al}$ respecto, Quintana y Aceituno (2006) señalan que existe una clara variabilidad interdecadal en el régimen de precipitación en Chile central, lo cual parece estar relacionado con los cambios en la intensidad del APSO, afectado por la PDO y el SOI. Así, las tendencias a la 
disminución de las precipitaciones coinciden con una significativa intensificación del APSO, un SOI positivo y una fase fría de la PDO. Por el contrario, un debilitamiento del anticiclón conlleva un aumento de las precipitaciones, fase negativa del SOI (Episodios El Niño) y una fase cálida de la PDO (Quintana y Aceituno, 2012). A partir de ello, se puede esperar un comportamiento decadal de los tipos de J\&C, es decir, intensificación de los tipos anticiclónicos (de 1950 a 1960, aproximadamente) y una disminución de estos tipos a mediados de la década de 1970.

Respecto a la variabilidad interanual de los tipos de J\&C, nos planteamos como hipótesis que la Oscilación Antártica está muy relacionada con la mayor frecuencia de tipos ciclónicos, sobre todo de los meses del invierno austral (abril a septiembre), lo cual coincidiría con lo señalado por Quintana y Aceituno (2012) respecto al aumento de las precipitaciones invernales.

Otras afirmaciones respecto a la circulación de Chile central son las de Romero (1985), el cual indica que:

- Chile central se caracteriza por la estabilidad de las condiciones anticiclónicas, las cuales alternan gradualmente con una derivación de las mismas: las depresiones térmicas de verano. Un tercio de los tipos de circulación son de naturaleza anticiclónica, mientras que el resto de situaciones son ciclónicas y advectivas;

- En términos generales, las situaciones anticiclónicas aumentan relativamente entre mayo y septiembre, producto de una reducción de los tipos advectivos en verano;

- La frecuencia de tipos ciclónicos es bastante regular en el año en el área comprendida entre los $28^{\circ}$ y $39^{\circ} \mathrm{S}$, excepción hecha del mes de noviembre, cuando decrecen relativamente;

- Los vientos son procedentes mayoritariamente del S y del SE. En invierno el flujo es del W, coincidiendo con la disminución de la alta y el traslado de las depresiones hacia el norte; $\mathrm{y}$

- Las situaciones ciclónicas se asocian a flujos del NW, N y NE, mientras que las anticiclónicas se relacionan con vientos de componente sur (SW, S y SE).

\section{METODOLOGÍA}

La clasificación sinóptica de $\mathrm{J} \& \mathrm{C}$ es un método automático que permite conocer el tipo de circulación atmosférica a partir de la presión atmosférica reducida al nivel del mar de un mínimo de 9 puntos (Figura 1), la cual para el presente trabajo fue obtenida, para el período 1950-2010, del proyecto NCEP/NCAR Reanalysis a una resolución diaria, y a las 18 UTC, sumando un total de 22.280 días y 200.520 datos de presión.

El proyecto NCEP/NCAR nació en 1991. En un primer momento su objetivo fue comprobar los cambios en el clima de la década anterior e implementar nuevas predicciones. Fue posible implementar este proyecto a partir de datos meteorológicos de tipo convencional, y a escala global (radiosondeos, datos de observatorios sobre superficies marinas, datos de aviones, datos sinópticos superficiales, datos de satélite, etc.). Dichos datos han sido pre-procesados para comprobar su calidad y la homogeneidad de las series, y se han obtenido diferentes datos de reanálisis de todas las variables meteorológicas y a distintos niveles (Kalnay et al., 1996).

La clasificación de J\&C consta de 27 tipos sinópticos: 8 advectivos puros (N, NE, E, SE, S, SW, W y NW), 1 ciclónico (C), 1 anticiclónico (A), 8 híbridos advectivo-ciclónicos (CN, 


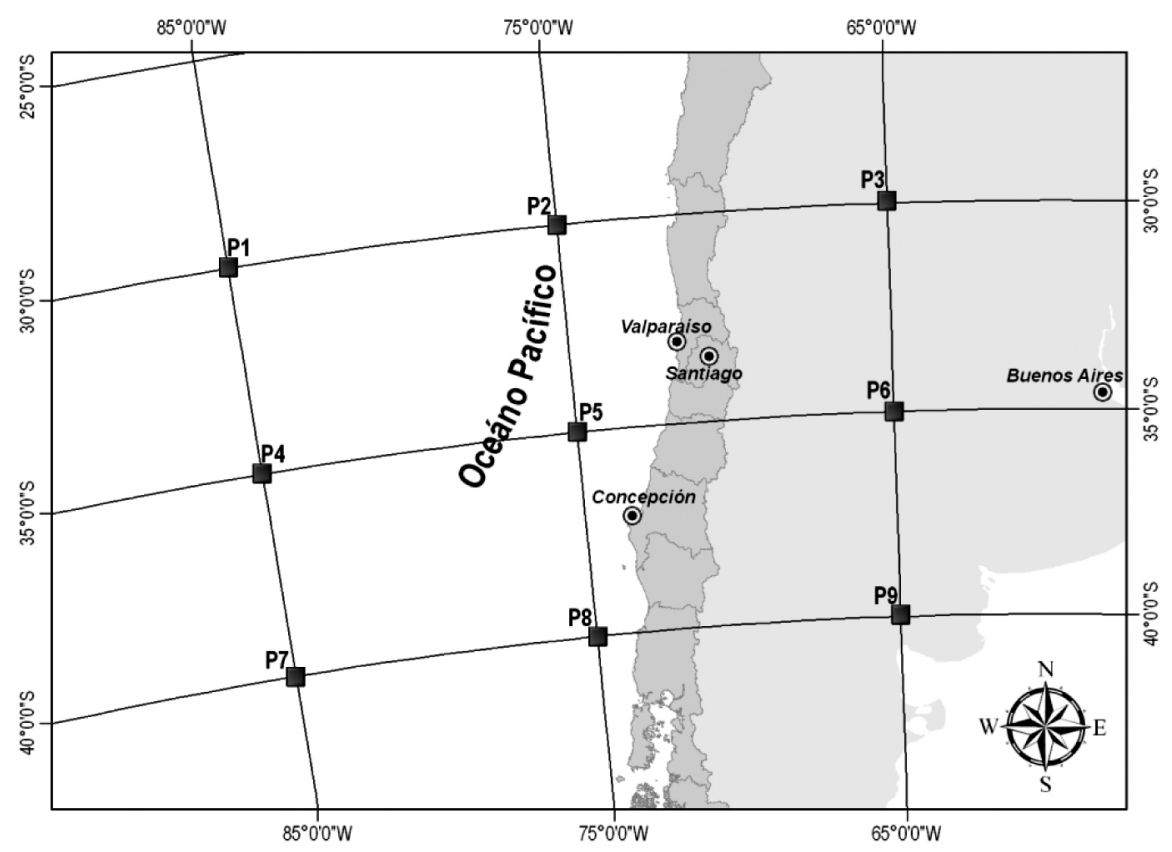

CNE, CE, CSE, CS, CSW, CW y CNW), 8 híbridos advectivo-anticiclónicos (AN, ANE, AE, ASE, AS, ASW, AW y ANW) y 1 indeterminado (U).

Las variables a calcular para la aplicación del método de $\mathrm{J} \& \mathrm{C}$ son la presión media reducida a nivel del mar (P), la componente zonal del viento geostrófico (W) (en nuestro caso entre los $30^{\circ}$ y $40^{\circ} \mathrm{S}$ ), la componente meridiana del viento geostrófico (S) (en nuestro caso entre los $85^{\circ}$ y $65^{\circ} \mathrm{W}$ ), la dirección del viento (D), la velocidad del viento en $\mathrm{m} / \mathrm{s}(\mathrm{F})$, la componente zonal de la vorticidad $\left(\mathrm{Z}_{\mathrm{W}}\right)$, la componente meridiana de la vorticidad $\left(\mathrm{Z}_{\mathrm{S}}\right)$ y la vorticidad total $(Z)$. Las expresiones analíticas ajustadas para Chile central son las siguientes:

$$
\begin{aligned}
& \mathrm{P}=0,0625[(\mathrm{P} 1+\mathrm{P} 3+\mathrm{P} 7+\mathrm{P} 9)+2(\mathrm{P} 2+\mathrm{P} 4+\mathrm{P} 6+\mathrm{P} 8)+4 \mathrm{P} 5] \\
& \mathrm{W}=0,25[(\mathrm{P} 1+2 \mathrm{P} 2+\mathrm{P} 3)-(\mathrm{P} 7+2 \mathrm{P} 8+\mathrm{P} 9)] \\
& \mathrm{S}=0,610[1 / 4(\mathrm{P} 1+2 \mathrm{P} 4+\mathrm{P} 7)-1 / 4(\mathrm{P} 3+2 \mathrm{P} 6+\mathrm{P} 9)] \\
& \mathrm{D}=\tan ^{-1}(\mathrm{~W} / \mathrm{S}) \\
& \mathrm{F}=\sqrt{ }\left(\mathrm{W}^{2}+\mathrm{S}^{2}\right) \\
& \mathrm{Zw}=1,068[(\mathrm{P} 1+2 \mathrm{P} 2+\mathrm{P} 3)-(\mathrm{P} 4+2 \mathrm{P} 5+\mathrm{P} 6)]-0,942 \times[(\mathrm{P} 4+2 \mathrm{P} 5+\mathrm{P} 6)-(\mathrm{P} 7+2 \mathrm{P} 8+\mathrm{P} 9)] \\
& \mathrm{Zs}=1,221[1 / 4(\mathrm{P} 1+2 \mathrm{P} 4+\mathrm{P} 7)-1 / 4(\mathrm{P} 2+2 \mathrm{P} 5+\mathrm{P} 8)-1 / 4(\mathrm{P} 2+2 \mathrm{P} 5+\mathrm{P} 8)+1 / 4(\mathrm{P} 3+2 \mathrm{P} 6+\mathrm{P} 9)] \\
& \mathrm{Z}=\mathrm{Zw}+\mathrm{Zs}
\end{aligned}
$$


A partir de los valores de las expresiones analíticas anteriores y siguiendo el método de $\mathrm{J} \& \mathrm{C}$ se aplican las siguientes 5 reglas:

1) La dirección del flujo viene dada por $\mathrm{D}$ (se usan 8 direcciones del viento, teniendo en cuenta el signo de W y S)

2) Si $|\mathrm{Z}|<\mathrm{F}$, existe un tipo advectivo o direccional puro, definido según la regla 1 (N, NE, E, SE, S, SW, W y NW)

3) Si $|Z|>2 F$, existe un tipo ciclónico (C) si $Z>0$, o anticiclónico (A) si $Z<0$

4) $\quad$ Si $F<|Z|<2 F$, existe un tipo híbrido, según el signo de $Z$ (regla 3 ) y la dirección del flujo obtenido de la regla 1 (CN, CNE, CE, CSE, CS, CSW, CW, CNW, AN, ANE, AE, ASE, AS, ASW, AW y ANW)

5) $\quad$ Si $\mathrm{F}<6$ o $|\mathrm{Z}|<6$, existe un tipo indeterminado (U)

De los 27 tipos de J\&C, el único tipo que no se encontró entre 1950 y 2010 fue el indeterminado (U), posiblemente por la marcada presencia del APSO y unos muy notables contrastes topográficos y térmicos de la superficie del área de estudio. Por lo tanto, se obtienen 26 tipos, los cuales además han sido juntados en tres diferentes agrupaciones:

- 10 grupos: A, C y los 8 rumbos del viento (con los tipos advectivos e híbridos), por ejemplo el Rumbo [N] corresponde al direccional N más los híbridos AN y CN. Y [W] agrupa al direccional W más los híbridos AW y CW.

- 5 grupos: A, C, ADVA (AN, ANE, AE, ASE, AS, ASW, AW y ANW), ADVC (CN, CNE, CE, CSE, CS, CSW, CW, CNW) y ADV (N, NE, E, SE, S, SW, W y NW).

- 3 grupos: ANT (A, AN, ANE, AE, ASE, AS, ASW, AW y ANW), CYC (C, CN, CNE, CE, CSE, CS, CSW, CW, CNW) y ADV (N, NE, E, SE, S, SW, W y NW).

El análisis de los tipos sinópticos se realizó para cuatro agregaciones temporales: Anual para toda la serie (1950-2010), anual en dos subperíodos (1950-1980 y 1981-2010), y a nivel mensual y estacional. La evaluación de las tendencias fue realizada con el software AnClim (Štěpánek, 2003), permitiendo así estimar los cambios en los tipos sinópticos de Jenkinson y Collison y en las otras agrupaciones. Para tal fin, se calcularon las tendencias para el período 1950-2010 (61 años), y se aplicaron test de normalidad y de significación estadística. Además, se compararon los subperíodos 1950-1980 (31 años) y 1981-2010 (30 años) para estimar si los cambios de tipos sinópticos han sido estadísticamente significativos, aplicándose el Test-Z de comparación de proporciones binomiales, y a un nivel de confianza del $95 \%$.

Un segundo objetivo fue evaluar si existen relaciones de los tipos sinópticos de J\&C con las teleconexiones que afectan a Chile central: El Niño Oscilación del Sur (mediante los índices $\mathrm{SOI}^{2}$ y $\left.\mathrm{MEI}^{3}\right)$, la Oscilación Decadal del Pacífico $\left(\mathrm{PDO}^{4}\right)$ y la Oscilación Antártica $\left(\mathrm{AAO}^{5}\right)$. Así, se correlacionaron estas teleconexiones con los tipos de J\&C. Además, se calculó a partir del flujo de viento obtenido mediante J\&C el índice del Oeste (Western Index, WI en siglas). Para la obtención del WI se agrupan las direcciones de los vientos en

\footnotetext{
2 Disponible en http://www.cru.uea.ac.uk/cru/data/soi/soi.dat

Disponible en http://www.esrl.noaa.gov/psd/enso/mei.ext/table.ext.html

Disponible en http://jisao.washington.edu/pdo/PDO.latest

Disponible en http://www.jisao.washington.edu/aao/slp/
} 
cuatro categorías: N, E (NE, E y SE), S y W (SW, W y NW), siendo WI la proporción de W para cada mes y año, por lo tanto, WI posee valores entre 0 y 1 . Para el Reino Unido, el WI no supera el valor 0,46 (Wheeler et al., 2010). Para Chile central el índice del Oeste fue, además, normalizado a nivel mensual para ver su variabilidad interanual (a modo de anomalías del índice), pues por lo general los meses de invierno arrojan valores más altos que el resto del año. Complementariamente se determinará si el WI posee un comportamiento cíclico, lo cual se lleva a cabo mediante el análisis espectral.

\section{RESULTADOS}

\section{Frecuencias anuales de los tipos sinópticos de Jenkinson \& Collison y sus tendencias}

Se observa de manera agregada un predominio de las circulaciones anticiclónicas para Chile central, lo cual se explica por la marcada persistencia del APSO. Con ello los tipos ANT alcanzan el 54\% de los días entre 1950 y 2010, seguidos de los tipos ADV, con un 31\%, y los CYC, con un $15 \%$. No obstante, hay tendencias significativas que indican la disminución de los tipos ANT (Tabla 1) y el aumento en los tipos CYC y ADV. Esto permite señalar que existen cambios en los tipos de circulación que afectan a Chile central.

Tabla 1

NÚMERO Y PORCENTAJE DE DIASS SEGÚN TIPOS SINÓPTICOS DE J\&C REAGRUPADOS SEGÚN EL SIGNO DE SU VORTICIDAD (ANT, CYC YADV) QUE POSEEN UNA VARIACIÓN SIGNIFICATIVA EN EL PERIODO 1950-2010 Y ENTRE LOS SUBPERIOODOS 1950-1980 Y 1981-2010 EN CHILE CENTRAL

\begin{tabular}{|l|c|c|c|c|r|}
\hline $\begin{array}{c}\text { Tipos de J\&C } \\
\text { reagrupados } \\
\text { por signo de } \\
\text { la vorticidad }\end{array}$ & $\begin{array}{c}\text { Número de } \\
\text { días (\%) } \\
\mathbf{( 1 9 5 0 - 2 0 1 0 )}\end{array}$ & $\begin{array}{c}\text { Número de } \\
\text { días }(\mathbf{\%}) \\
\mathbf{( 1 9 5 0 - 1 9 8 0 )}\end{array}$ & $\begin{array}{c}\text { Número de } \\
\text { días (\%) } \\
\mathbf{( 1 9 8 1 - 2 0 1 0 )}\end{array}$ & $\begin{array}{c}\text { Aumenta } \\
\text { / Dismi- } \\
\text { nuye }\end{array}$ & $\begin{array}{c}\text { p-valor de la } \\
\text { tendencia entre } \\
\text { los subperíodos }\end{array}$ \\
\hline ANT & $12.135(54,5 \%)$ & $6.517(57,6 \%)$ & $5.618(51,3 \%)$ & Disminuye & 0,000 \\
\hline CYC & $3.257(14,6 \%)$ & $1.561(13,8 \%)$ & $1.696(15,5 \%)$ & Aumenta & 0,085 \\
\hline ADV & $6.888(30,9 \%)$ & $3.245(28,7 \%)$ & $3.643(33,2 \%)$ & Aumenta & 0,000 \\
\hline
\end{tabular}

Diferentes tests de significación estadística (t de Student, Spearman y Mann Kendall) y de normalidad (Kolmogorov-Smirnov) han sido aplicados para determinar las tendencias lineales de las tres categorías analizadas anteriormente durante el período completo (Tabla 2), mostrando que los días de tipo ANT se han reducido en cerca de 10 días por década, los de tipo CYC han aumentado en algo más de tres, y los advectivos lo han hecho en casi 6 días por década.

Diferenciando los tipos híbridos anticiclónicos (ADVA) del anticiclónico (A) y de los advectivos (ADV), en la Tabla 3 se observa que el predominio del tipo A ya no es tan evidente para toda la serie. Sí lo fue entre 1950 y 1980, pero su presencia se ve reducida en un $7,8 \%$ en el treintenio final, a costa de los tipos advectivos puros, que pasan de un $28,7 \%$ a un $33,2 \%$ de manera estadísticamente significativa. Así, el tipo A decrece un 1,5\% más que los ANT de la Tabla 1, que agrupa los anticiclónicos y los híbridos anticiclónicos. 
Tabla 2

TENDENCIAS LINEALES SIGNIFICATIVAS $(A=0,05)$ PARA LOS TIPOS SINÓPTICOS DE J\&C AGRUPADOS POR EL SIGNO DE LA VORTICIDAD (ANT, CYC YADV), EN EL PERIODO 1950-2010

\begin{tabular}{|c|c|c|c|c|}
\hline $\begin{array}{c}\text { Tipos de J\&C } \\
\text { reagrupados por } \\
\text { signo de la vor- } \\
\text { ticidad }\end{array}$ & t, Sp y MK (1) & $\begin{array}{c}\text { Test de norma- } \\
\text { lidad (2) D } \\
\mathrm{p} \text {-valor }\end{array}$ & $\begin{array}{c}\text { Tendencia días/ } \\
\text { década }\end{array}$ & Regresión lineal \\
\hline ANT & t, Sp y MK & 0,$059 ; 0,984$ & $-8,90$ días/década & $\begin{array}{c}\mathrm{y}=-0,89 \mathrm{x}+ \\
226,53\end{array}$ \\
\hline CYC & $\mathrm{t}, \mathrm{Sp}$ y MK & 0,$091 ; 0,694$ & $+3,15$ días/ década & $\mathrm{y}=0,32 \mathrm{x}+43,63$ \\
\hline $\mathrm{ADV}$ & $\mathrm{t}, \mathrm{Sp}$ y MK & 0,$090 ; 0,706$ & $+5,75$ días/ década & $\mathrm{y}=0,58 \mathrm{x}+95,08$ \\
\hline
\end{tabular}

(1) Significación estadística mediante el test t-Student (t), rangos de Spearman (Sp) y Mann Kendall (MK).

(2) Test de Kolmogorov-Smirnov.

Tabla 3

NÚMERO Y PORCENTAJE DE DÍAS SEGÚN TIPOS SINÓPTICOS DE J\&C REAGRUPADOS ENA, C, ADVA, ADVC Y ADV, Y SUS TENDENCIAS EN EL PERÍODO 1950-2010 Y ENTRE LOS SUBPERÍODOS 1950-1980 Y 1981-2010

\begin{tabular}{|c|c|c|c|c|c|}
\hline $\begin{array}{c}\text { Tipos de J\&C } \\
\text { reagrupados }\end{array}$ & $\begin{array}{c}\text { Número de días } \\
(\%)(\mathbf{1 9 5 0 - 2 0 1 0})\end{array}$ & $\begin{array}{c}\text { Número de } \\
\text { días (\%) (1950- } \\
\mathbf{1 9 8 0})\end{array}$ & $\begin{array}{c}\text { Número de } \\
\text { días (\%) } \\
(\mathbf{1 9 8 1 - 2 0 1 0})\end{array}$ & $\begin{array}{c}\text { Aumenta } \\
/ \text { Dismi- } \\
\text { nuye }\end{array}$ & p-valor(*) \\
\hline A & $7.252(32,6 \%)$ & $4.126(36,4 \%)$ & $3.126(28,6 \%)$ & $\begin{array}{c}\text { Dismi- } \\
\text { nuye }\end{array}$ & 0,000 \\
\hline C & $1.813(8,1 \%)$ & $857(7,6 \%)$ & $956(8,7 \%)$ & Aumenta & 0,196 \\
\hline ADVA & $4.883(21,9 \%)$ & $2.391(21,1 \%)$ & $2.492(22,7 \%)$ & Aumenta & 0,088 \\
\hline ADVC & $1.444(6,5 \%)$ & $704(6,2 \%)$ & $740(6,8 \%)$ & Aumenta & 0,322 \\
\hline ADV & $6.888(30,9 \%)$ & $3.245(28,7 \%)$ & $3.643(33,2 \%)$ & Aumenta & 0,000 \\
\hline
\end{tabular}

(*):p-valor de la tendencia entre los subperíodos.

Tabla 4

TENDENCIAS LINEALES SIGNIFICATIVAS $(A=0,05)$ PARA LOS TIPOS SINÓPTICOS DE J\&C REAGRUPADOS ENA, C, ADVA, ADVC Y ADV, EN EL PERIOODO 1950-2010

\begin{tabular}{|c|c|c|c|c|}
\hline $\begin{array}{c}\text { Tipos de J\&C } \\
\text { reagrupados }\end{array}$ & t, Sp y MK (1) & $\begin{array}{c}\text { Test de norma- } \\
\text { lidad (2) D ; } \\
\text { p-valor }\end{array}$ & $\begin{array}{c}\text { Tendencia días/ } \\
\text { década }\end{array}$ & Regresión lineal \\
\hline A & t, Sp y MK & 0,$068 ; 0,940$ & $-10,45$ días / década & $\mathrm{y}=-1,04 \mathrm{x}+151,27$ \\
\hline $\mathrm{C}$ & $\mathrm{t}, \mathrm{Sp}$ y MK & 0,$063 ; 0,967$ & $+2,22$ días década & $\mathrm{y}=0,22 \mathrm{x}+22,85$ \\
\hline ADVA & t, Sp y MK & 0,$060 ; 0,981$ & $+1,55$ días / década & $\mathrm{y}=0,15 \mathrm{x}+75,26$ \\
\hline ADVC & Sp y MK & 0,$081 ; 0,819$ & $+0,932$ días / década & $\mathrm{y}=0,93 \mathrm{x}+20,78$ \\
\hline ADV & $\mathrm{t}$, Sp y MK & 0,$090 ; 0,706$ & $+5,75$ días / década & $\mathrm{y}=0,58 \mathrm{x}+95,08$ \\
\hline
\end{tabular}




\section{Figura 2}

FRECUENCIA DE LOS TIPOS SINÓPTICOS J\&C REDUCIDOS A 10 CATEGORÍAS SEGÚN [RUMBOS] PARA CHILE CENTRAL, ENTRE 1950 Y 2010

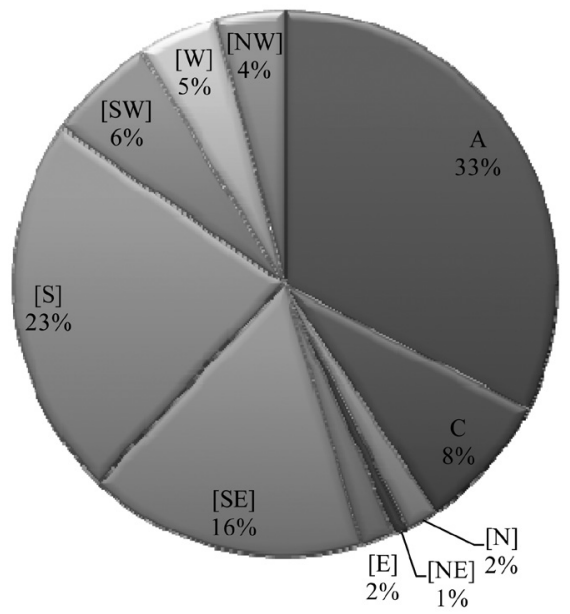

$\mathrm{Al}$ reducir los tipos de $\mathrm{J} \& \mathrm{C}$ a 10 categorías según rumbos (Fig. 2) los tipos direccionales aumentan su dominio con un 59\%, destacando principalmente los direccionales del [S] (23\%) y del [SE] (16\%). Un hecho a destacar es que las circulaciones anticiclónicas corresponden a un 33\% de los tipos de tiempo de Chile central, algo ya advertido por Romero (1985). No obstante, los días anticiclónicos han reducido su importancia en los últimos 30 años (Tabla 5), desde un 36,4\% en el primer subperíodo a un 28,6\% en el segundo, una caída ya advertida de un $7,8 \%$, tal como se dijo anteriormente. Por el contrario, el tipo [S] ha crecido un 4,7\%, desde un 20,4\% en el subperíodo $1950-1980$ a un 25,1\% en el 1981-2010.

Tabla 5

FRECUENCIA Y PORCENTAJE DE LOS TIPOS SINÓPTICOS J\&C REDUCIDOS A 10 CATEGORIAS SEGÚN [RUMBOS] PARA CHILE CENTRAL EN LOS SUBPERIODOS 1950-1980 Y 1981-2010

\begin{tabular}{|l|c|c|}
\hline $\begin{array}{l}\text { Tipos J\&C según } \\
\text { [rumbos] }\end{array}$ & $\begin{array}{c}\text { Número de días (\%) } \\
(\mathbf{1 9 5 0 - 1 9 8 0 )}\end{array}$ & $\begin{array}{c}\text { Número de días (\%) } \\
(\mathbf{1 9 8 1 - 2 0 1 0 )}\end{array}$ \\
\hline Anticiclónicos (A) & $4.126(36,4 \%)$ & $3.126(28,5 \%)$ \\
\hline Ciclónicos (C) & $857(7,6 \%)$ & $956(8,7 \%)$ \\
\hline$[\mathrm{N}]$ & $189(1,7 \%)$ & $233(2,1 \%)$ \\
\hline$[\mathrm{NE}]$ & $86(0,8 \%)$ & $94(0,9 \%)$ \\
\hline$[\mathrm{E}]$ & $233(2,1 \%)$ & $232(2,1 \%)$ \\
\hline$[\mathrm{SE}]$ & $2.017(17,8 \%)$ & $1.655(15,1 \%)$ \\
\hline$[\mathrm{S}]$ & $2.315(20,4)$ & $2.745(25,1 \%)$ \\
\hline$[\mathrm{SW}]$ & $614(5,4 \%)$ & $841(7,7 \%)$ \\
\hline$[\mathrm{W}]$ & $486(4,3 \%)$ & $572(5,2 \%)$ \\
\hline$[\mathrm{NW}]$ & $400(3,5 \%)$ & $503(4,6 \%)$ \\
\hline
\end{tabular}


Tabla 6

NÚMERO Y PORCENTAJE DE DÍAS, DESVIACIÓN ESTÁNDAR, MÍNIMOS Y MÁXIMOS, COEFICIENTE DE VARIACIÓN E INDICE DE IRREGULARIDAD TEMPORAL DE LOS TIPOS DE J\&C DEL PERÍODO 1950-2010 EN CHILE CENTRAL

\begin{tabular}{|c|c|c|c|c|c|c|c|c|}
\hline $\begin{array}{l}\text { Tipos } \\
\text { J\&C }\end{array}$ & Total & $\begin{array}{l}\text { Promedio } \\
\text { días al año }\end{array}$ & $\begin{array}{c}\text { Porcentaje } \\
(\%)\end{array}$ & Máximo & Mínimo & $\begin{array}{c}\text { Desv. } \\
\text { Estándar }\end{array}$ & $\begin{array}{c}\text { Coef. de } \\
\text { variación }\end{array}$ & $\begin{array}{c}\text { Índice de } \\
\text { irregularidad } \\
\text { temporal }\end{array}$ \\
\hline A & 7.252 & 118,89 & $32,55 \%$ & 176 & 62 & 27,77 & 0,234 & 0,143 \\
\hline ANE & 18 & 0,30 & $0,08 \%$ & 2 & 0 & 0,56 & 1,891 & 0,023 \\
\hline $\mathrm{AE}$ & 66 & 1,08 & $0,30 \%$ & 5 & 0 & 1,19 & 1,097 & 0,268 \\
\hline ASE & 763 & 12,51 & $3,42 \%$ & 24 & 3 & 5,22 & 0,417 & 0,348 \\
\hline AS & 2.272 & 37,25 & $10,20 \%$ & 54 & 23 & 7,60 & 0,204 & 0,224 \\
\hline ASW & 833 & 13,66 & $3,74 \%$ & 26 & 2 & 4,87 & 0,357 & 0,329 \\
\hline AW & 517 & 8,48 & $2,32 \%$ & 17 & 0 & 3,83 & 0,452 & 0,392 \\
\hline ANW & 338 & 5,54 & $1,52 \%$ & 11 & 1 & 2,51 & 0,452 & 0,483 \\
\hline AN & 76 & 1,25 & $0,34 \%$ & 4 & 0 & 1,16 & 0,934 & 0,253 \\
\hline $\mathrm{NE}$ & 85 & 1,39 & $0,38 \%$ & 4 & 0 & 1,08 & 0,778 & 0,318 \\
\hline $\mathrm{E}$ & 245 & 4,02 & $1,10 \%$ & 14 & 0 & 2,73 & 0,680 & 0,683 \\
\hline $\mathrm{SE}$ & 2.274 & 37,28 & $10,21 \%$ & 69 & 15 & 10,69 & 0,287 & 0,263 \\
\hline $\mathrm{S}$ & 2.546 & 41,74 & $11,43 \%$ & 76 & 16 & 13,54 & 0,324 & 0,241 \\
\hline SW & 539 & 8,84 & $2,42 \%$ & 19 & 1 & 4,34 & 0,492 & 0,593 \\
\hline W & 479 & 7,85 & $2,15 \%$ & 17 & 1 & 3,39 & 0,431 & 0,564 \\
\hline NW & 474 & 7,77 & $2,13 \%$ & 20 & 2 & 3,40 & 0,438 & 0,440 \\
\hline $\mathrm{N}$ & 246 & 4,03 & $1,10 \%$ & 13 & 0 & 2,46 & 0,611 & 0,557 \\
\hline $\mathrm{C}$ & 1.813 & 29,72 & $8,14 \%$ & 51 & 10 & 9,63 & 0,324 & 0,299 \\
\hline CNE & 77 & 1,26 & $0,35 \%$ & 6 & 0 & 1,18 & 0,936 & 0,221 \\
\hline $\mathrm{CE}$ & 154 & 2,52 & $0,69 \%$ & 8 & 0 & 1,69 & 0,669 & 0,485 \\
\hline CSE & 635 & 10,41 & $2,85 \%$ & 23 & 2 & 4,62 & 0,444 & 0,486 \\
\hline $\mathrm{CS}$ & 242 & 3,97 & $1,09 \%$ & 11 & 0 & 2,46 & 0,621 & 0,625 \\
\hline CSW & 83 & 1,36 & $0,37 \%$ & 8 & 0 & 1,48 & 1,090 & 0,279 \\
\hline $\mathrm{CW}$ & 62 & 1,02 & $0,28 \%$ & 4 & 0 & 0,90 & 0,889 & 0,129 \\
\hline $\mathrm{CNW}$ & 91 & 1,49 & $0,41 \%$ & 5 & 0 & 1,23 & 0,827 & 0,296 \\
\hline $\mathrm{CN}$ & 100 & 1,64 & $0,45 \%$ & 7 & 0 & 1,74 & 1,063 & 0,349 \\
\hline
\end{tabular}

Con los 22.280 días, clasificados en los 26 tipos sinópticos de Jenkinson y Collison encontrados para Chile central, se obtiene que los tipos más frecuentes son el tipo A, con 7.252 días $(32,55 \%)$, S, con 2.546 días $(11,43 \%)$, SE, con 2.274 días $(10,21 \%)$ y AS, con 2.272 días (10,20\%) (Tabla 6). A ellos le siguen en importancia, con menos de 2.000 días, el tipo C (8,14\%), ASW (3,74\%), ASE (3,42\%), CSE $(2,85 \%)$ y SW (2,42\%). La suma de estos 9 tipos sinópticos supone el 85\% de todos los días del período 1950-2010. 
Tabla 7

TENDENCIAS, DISTRIBUCIÓN NORMAL, SIGNIFICACIÓN ESTADISTIICA Y REGRESIÓN LINEAL DE LOS TIPOS DE J\&C, PARA EL PERIOODO 1950-2010 EN CHILE CENTRAL

\begin{tabular}{|c|c|c|c|c|}
\hline Tipos J\&C & $\begin{array}{r}\text { Tendenc } \\
\text { déc: }\end{array}$ & $\begin{array}{l}\text { la días/ } \\
\text { da }\end{array}$ & $\begin{array}{l}\text { Sig. estadís- } \\
\text { tica }^{(1)}\end{array}$ & Regresión lineal \\
\hline A & $-10,45$ & $\sqrt{ }$ & $\mathrm{t}, \mathrm{Sp}$ y $\mathrm{MK}$ & $Y=-1,045 x+151,270$ \\
\hline ANE & $+0,02$ & & MK & $Y=0,002 x+0,245$ \\
\hline $\mathrm{AE}$ & $-0,16$ & & MK & $Y=-0,016 x+1,579$ \\
\hline ASE & $-1,18$ & $\sqrt{ }$ & t, Sp y MK & $Y=-0,118 x+16,157$ \\
\hline AS & $+1,22$ & $\sqrt{ }$ & t y $\mathrm{MK}$ & $Y=0,122 x+33,452$ \\
\hline ASW & $+1,52$ & $\sqrt{ }$ & t, Sp y MK & $Y=0,152 x+8,931$ \\
\hline AW & $+0,46$ & $\sqrt{ }$ & $\mathrm{Sp}$ & $Y=0,046 x+7,039$ \\
\hline ANW & $-0,23$ & $\sqrt{ }$ & MK & $Y=-0,023 x+6,244$ \\
\hline AN & $-0,12$ & & MK & $Y=-0,012 x+1,607$ \\
\hline NE & $+0,01$ & & MK & $Y=0,001 x+1,364$ \\
\hline $\mathrm{E}$ & $+0,08$ & & no sig. & $Y=0,007 x+3,785$ \\
\hline SE & $-1,22$ & $\sqrt{ }$ & MK & $Y=-0,122 x+41,074$ \\
\hline S & $+4,34$ & $\sqrt{ }$ & $\mathrm{t}, \mathrm{Sp}$ y $\mathrm{MK}$ & $Y=0,434 x+28,285$ \\
\hline SW & $+1,06$ & $\sqrt{ }$ & t, Sp y MK & $Y=0,106 x+5,559$ \\
\hline $\mathrm{W}$ & $+0,21$ & & no sig. & $Y=0,021 x+7,211$ \\
\hline NW & $+0,73$ & $\sqrt{ }$ & t, Sp y MK & $Y=0,072 x+5,523$ \\
\hline $\mathrm{N}$ & $+0,57$ & & t y Sp & $Y=0,056 x+2,282$ \\
\hline $\mathrm{C}$ & $+2,22$ & $\sqrt{ }$ & t, Sp y MK & $Y=0,221 x+22,854$ \\
\hline CNE & $+0,04$ & & no sig. & $Y=0,004 x+1,141$ \\
\hline $\mathrm{CE}$ & $-0,11$ & $\sqrt{ }$ & MK & $Y=-0,011 x+2,874$ \\
\hline CSE & $+0,11$ & $\sqrt{ }$ & no sig. & $Y=0,013 x+9,998$ \\
\hline CS & $+0,57$ & & t y Sp & $Y=0,057 x+2,188$ \\
\hline CSW & $+0,14$ & & $\mathrm{Sp}$ & $Y=0,014 x+0,925$ \\
\hline $\mathrm{CW}$ & $+0,04$ & & MK & $Y=0,004 x+0,892$ \\
\hline $\mathrm{CNW}$ & $+0,08$ & & MK & $Y=0,008 x+1,243$ \\
\hline $\mathrm{CN}$ & $+0,04$ & & no sig. & $Y=0,004 x+1,521$ \\
\hline
\end{tabular}

( $\sqrt{ })$ Distribución normal según test de Kolmogorov-Smirnov.

(1) Significación estadística mediante los test de t-Student (t), rangos de Spearman (Sp) y Mann Kendall (MK). 
Los cinco tipos más frecuentes presentan los siguientes máximos anuales en la serie: A con 176 días en 1953, S con 76 días en 2009, SE con 69 días en 1968, AS con 54 días en 1961, 1971 y 2003, y C con 51 días en 2000. El resto de los tipos J\&C nunca superaron los 26 días al año. Al revisar los tipos menos frecuentes, se advierte que hay 14 que en algunos años no se han presentado, cuatro de ellos híbridos anticiclónicos (ANE, AE, AW, AN), dos advectivos (E y N) y siete híbridos ciclónicos (CNE, CE, CS, CW, CSW, CW, CNW, CN). Por otra parte, el coeficiente de variación mínimo es de un $20 \%$, encontrándose que los mayores coeficientes corresponden a los tipos del norte y el este, que superan el 60\%. Además, el índice de irregularidad temporal $\left(\mathrm{S}_{1}\right.$, propuesto por Martín-Vide et al., 2001), y que expresa el promedio de los valores absolutos de los logaritmos neperianos de los cocientes de cada valor con el precedente (en este caso, días en el año en que se presenta cada tipo de J\&C), muestra sus mayores valores coincidentes con aquellos que presentaron un coeficiente de variación entre $44 \%$ y $68 \%$. No obstante, sobre dichos valores, el índice de irregularidad temporal es más eficiente, pues representa mejor lo que ocurre entre años, especialmente para tipos con una baja frecuencia, y justamente de componente norte, noreste y este (ANE, AE, AN, NE, CNE). Por ejemplo, el caso menos frecuente (ANE) se presentó en toda la serie en sólo 18 días, y el coeficiente de variación alcanzó un muy elevado 189\%, mientras que el índice de irregularidad temporal fue de un 0,23 , valor medio entre su rango de valores.

Respecto a la tendencia de los tipos J\&C (Tabla 7), destaca la disminución del tipo A, con una pérdida por década de unos 10,45 días (64,8 días en todo el período). Dicha disminución ha sido acompañada por el aumento de los tipos S (4,34 días/década), C (2,22 días/década) y ASW (1,52 días/década).

\section{Frecuencias mensuales y tendencias estacionales según los tipos de Jenkinson \& Collison}

Respecto a la variabilidad intranual y siguiendo el método de J\&C, son 16 tipos sinópticos los que cubren el $96 \%$ de los días. Las condiciones anticiclónicas son más importantes entre mayo y noviembre (más del 30\% de los días), y poseen un mínimo en febrero, posiblemente relacionado con bajas térmicas, pues coincide con el mes de verano con más situaciones ciclónicas. Las circulaciones del sur y sureste, que conllevan buen tiempo, son frecuentes entre los meses de octubre y abril, mientras que las circulaciones de mal tiempo (suroeste, oeste y noroeste) son características del período abril-octubre (Figura 3).

Las circulaciones atmosféricas de Chile central más representativas son las A, C, AS, AW, SE y S (Fig. 4), y las menos frecuentes son las de tipo híbrido, específicamente las AN, ANE, AE, CN, CNE, CE, CSW, CW, CNW y las de tipo advectivo del NE. Ello indica la poca influencia de las circulaciones anticiclónicas, ciclónicas y de tipo advectivo con vientos norte, nordeste y este, y situaciones ciclónicas del oeste y noroeste.

Ahora reduciendo los tipos de J\&C a advectivos puros (ADV), advectivos anticiclónicos (ADVA) y advectivos ciclónicos (ADVC) (Fig. 5), tenemos que los advectivos puros y anticiclónicos son las circulaciones más frecuentes del verano, con máximos entre octubre y enero. Mientras que los advectivos ciclónicos (ADVC) muestran dos máximos en el año muy bien definidos, uno centrado entre marzo y abril, y el otro en el mes de julio. Los meses con menos días ADVC son enero y noviembre, lo cual en parte es similar a lo descrito por Romero (1985), cuando señalaba que en noviembre hay una disminución relativa de las circulaciones ciclónicas, en este caso de carácter ciclónico-advectivo. 
Figura 3

FRECUENCIA MENSUAL DE LOS TIPOS SINÓPTICOS DE JENKINSON \& COLLISONA, S, SE, C, SW, W Y NW PARA CHILE CENTRAL, ENTRE 1950 Y 2010

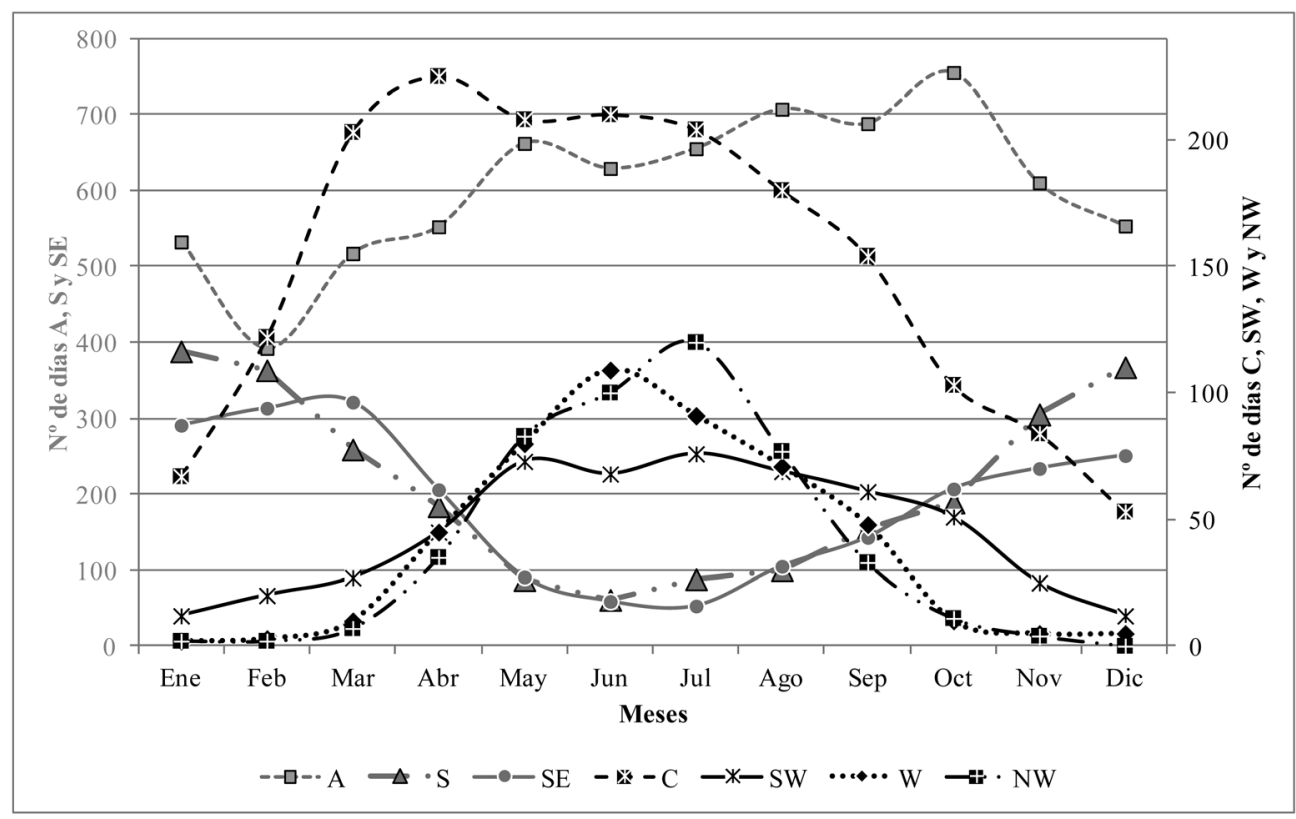

Figura 4

FRECUENCIA MENSUAL DE LOS TIPOS SINÓPTICOS DE J\&C PARA CHILE CENTRAL, ENTRE 1950 Y 2010

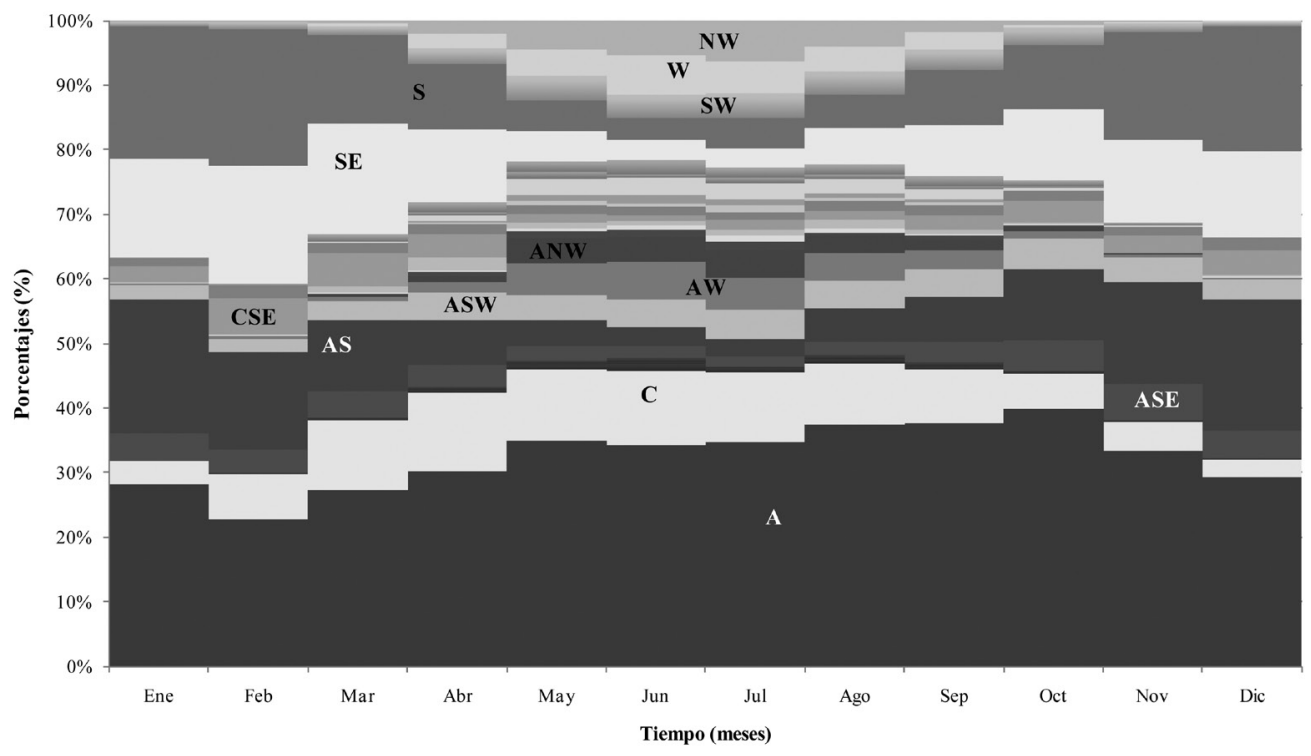

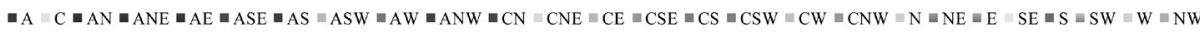


Figura 5

FRECUENCIA MENSUAL DE LOS TIPOS SINÓPTICOS DE JENKINSON \& COLLISONADVECTIVOS PUROS (ADV), ADVECTIVOS ANTICICLÓNICOS (ADVA) YADVECTIVOS CICLÓNICOS (ADVC) PARA CHILE CENTRAL, ENTRE 1950 Y 2010

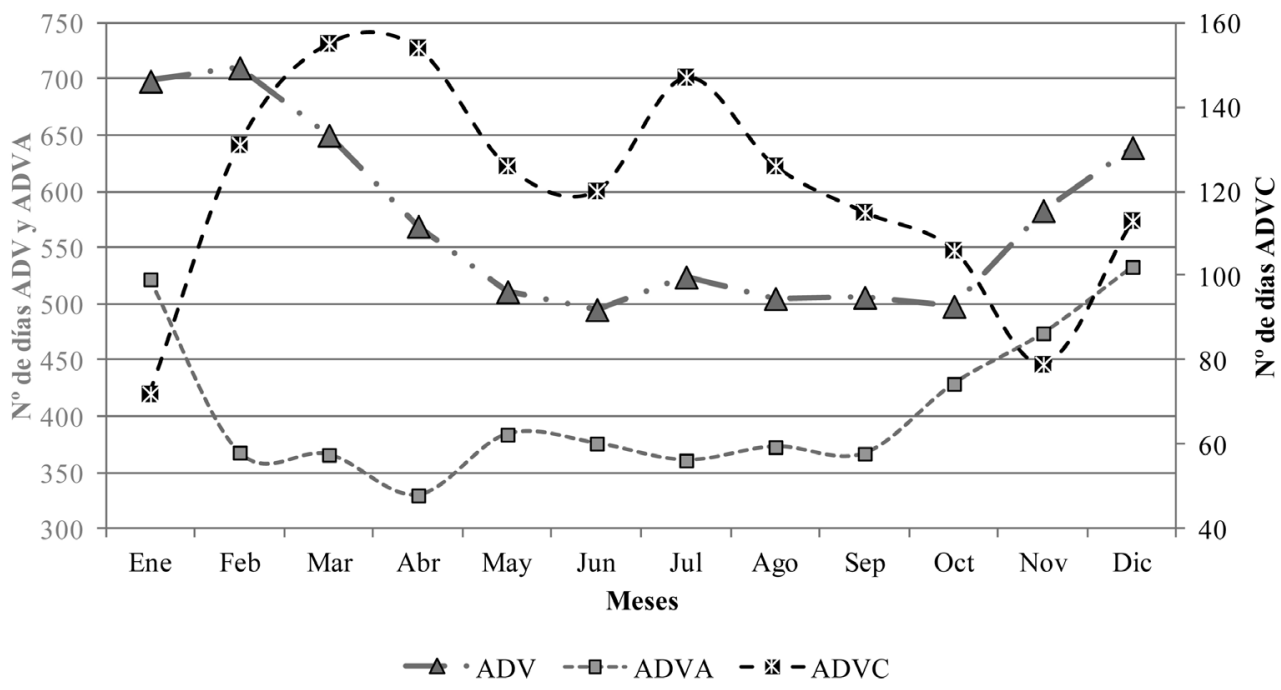

Figura 6

FRECUENCIA ESTACIONAL DEL RÉGIMEN DE VIENTOS OBTENIDO A PARTIR DE J\&C PARA CHILE CENTRAL, ENTRE 1950 Y 2010
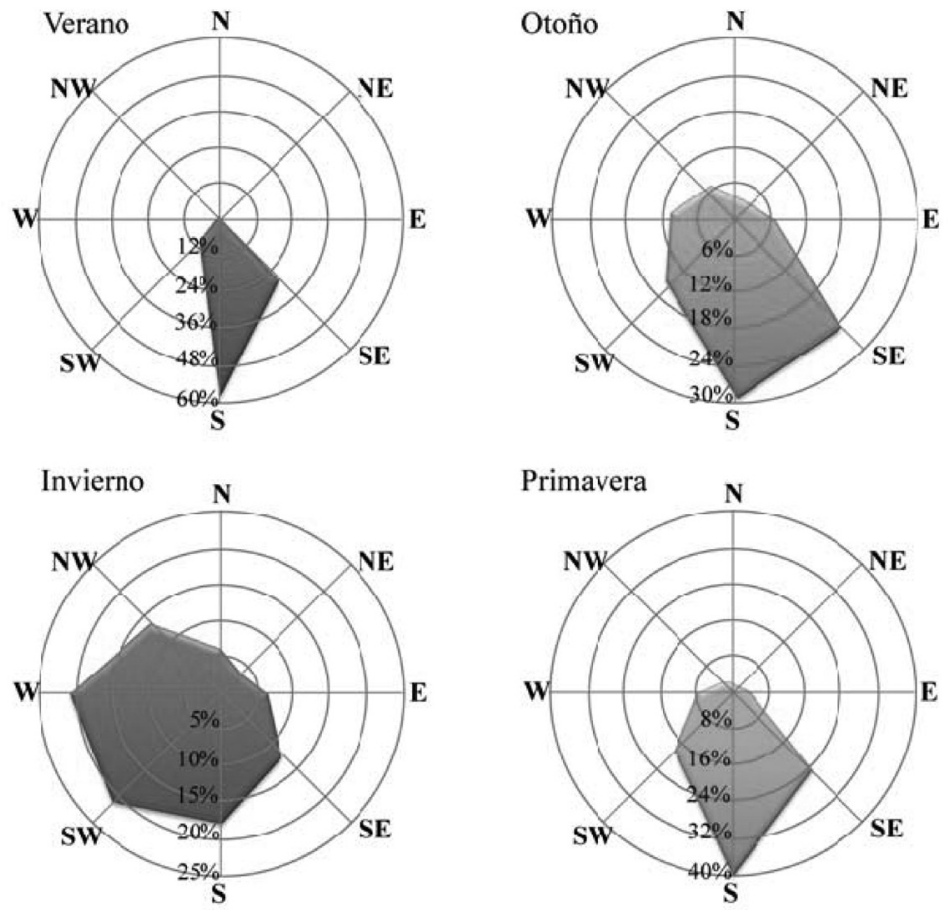
Variabilidad y tendencias climáticas en Chile central en el período 1950-2010..

Tabla 7

NÚMERO Y PORCENTAJE DE DÍAS SEGÚN TIPOS SINÓPTICOS DE J\&C MÁS FRECUENTES Y SUS TENDENCIAS EN LOS SUBPERIODOS 1950-1980 Y 1981-2010 PARA CHILE CENTRAL

\begin{tabular}{|c|c|c|c|c|c|c|c|}
\hline $\begin{array}{l}\text { Tipos } \\
\text { J\&C }\end{array}$ & Estación & $\begin{array}{c}N^{0} \text { de días } \\
(1950-1980)\end{array}$ & $\begin{array}{c}\mathrm{N}^{0} \text { de días } \\
(\mathbf{1 9 8 1 - 2 0 1 0 )}\end{array}$ & $\begin{array}{c}\% \\
(1950-1980)\end{array}$ & $\begin{array}{c}\% \\
(1981-2010) \\
\end{array}$ & $\begin{array}{c}\text { Aumenta / } \\
\text { Disminuye }\end{array}$ & p-valor \\
\hline \multirow{4}{*}{ A } & Verano & 830 & 647 & $56,19 \%$ & $43,81 \%$ & Disminuye & 0,000 \\
\hline & Otoño & 984 & 747 & $56,85 \%$ & $43,15 \%$ & Disminuye & 0,000 \\
\hline & Invierno & 1.133 & 858 & $56,91 \%$ & $43,09 \%$ & Disminuye & 0,000 \\
\hline & Primavera & 1.179 & 874 & $57,43 \%$ & $42,57 \%$ & Disminuye & 0,000 \\
\hline \multirow{4}{*}{ AS } & Verano & 484 & 546 & $46,99 \%$ & $53,01 \%$ & Aumenta & 0,027 \\
\hline & Otoño & 217 & 192 & $53,06 \%$ & $46,94 \%$ & Disminuye & 0,108 \\
\hline & Invierno & 103 & 99 & $50,99 \%$ & $49,01 \%$ & Disminuye & 0,611 \\
\hline & Primavera & 298 & 333 & $47,23 \%$ & $52,77 \%$ & Aumenta & 0,082 \\
\hline \multirow{4}{*}{ ASE } & Verano & 142 & 86 & $62,28 \%$ & $37,72 \%$ & Disminuye & 0,000 \\
\hline & Otoño & 113 & 76 & $59,79 \%$ & $40,21 \%$ & Disminuye & 0,004 \\
\hline & Invierno & 45 & 53 & $45,92 \%$ & $54,08 \%$ & Aumenta & 0,210 \\
\hline & Primavera & 140 & 108 & $56,45 \%$ & $43,55 \%$ & Disminuye & 0,022 \\
\hline \multirow{4}{*}{ ASW } & Verano & 53 & 89 & $37,32 \%$ & $62,68 \%$ & Aumenta & 0,002 \\
\hline & Otoño & 78 & 128 & $37,86 \%$ & $62,14 \%$ & Aumenta & 0,000 \\
\hline & Invierno & 120 & 127 & $48,58 \%$ & $51,42 \%$ & Aumenta & 0,328 \\
\hline & Primavera & 102 & 136 & $42,86 \%$ & $57,14 \%$ & Aumenta & 0,015 \\
\hline \multirow{4}{*}{$\mathrm{C}$} & Verano & 123 & 119 & $50,83 \%$ & $49,17 \%$ & Disminuye & 0,398 \\
\hline & Otoño & 288 & 348 & $45,28 \%$ & $54,72 \%$ & Aumenta & 0,009 \\
\hline & Invierno & 290 & 304 & $48,82 \%$ & $51,18 \%$ & Aumenta & 0,283 \\
\hline & Primavera & 156 & 185 & $45,75 \%$ & $54,25 \%$ & Aumenta & 0,059 \\
\hline \multirow{4}{*}{ CSE } & Verano & 113 & 101 & $52,80 \%$ & $47,20 \%$ & Disminuye & 0,207 \\
\hline & Otoño & 90 & 102 & $46,88 \%$ & $53,13 \%$ & Aumenta & 0,194 \\
\hline & Invierno & 34 & 37 & $47,89 \%$ & $52,11 \%$ & Aumenta & 0,361 \\
\hline & Primavera & 92 & 66 & $58,23 \%$ & $41,77 \%$ & Disminuye & 0,021 \\
\hline \multirow{4}{*}{ S } & Verano & 470 & 648 & $42,04 \%$ & $57,96 \%$ & Aumenta & 0,000 \\
\hline & Otoño & 233 & 297 & $43,96 \%$ & $56,04 \%$ & Aumenta & 0,003 \\
\hline & Invierno & 124 & 125 & $49,80 \%$ & $50,20 \%$ & Aumenta & 0,475 \\
\hline & Primavera & 281 & 368 & $43,30 \%$ & $56,70 \%$ & Aumenta & 0,000 \\
\hline \multirow{4}{*}{ SE } & Verano & 486 & 369 & $56,84 \%$ & $43,16 \%$ & Disminuye & 0,000 \\
\hline & Otoño & 359 & 259 & $58,09 \%$ & $41,91 \%$ & Disminuye & 0,000 \\
\hline & Invierno & 104 & 113 & $47,93 \%$ & $52,07 \%$ & Aumenta & 0,271 \\
\hline & Primavera & 299 & 285 & $51,20 \%$ & $48,80 \%$ & Disminuye & 0,719 \\
\hline \multirow{4}{*}{ SW } & Verano & 18 & 26 & $40,91 \%$ & $59,09 \%$ & Aumenta & 0,118 \\
\hline & Otoño & 53 & 92 & $36,55 \%$ & $63,45 \%$ & Aumenta & 0,001 \\
\hline & Invierno & 99 & 114 & $46,48 \%$ & $53,52 \%$ & Aumenta & 0,153 \\
\hline & Primavera & 54 & 83 & $39,42 \%$ & $60,58 \%$ & Aumenta & 0,008 \\
\hline
\end{tabular}


Lo anterior también deja sus efectos sobre el régimen de los vientos agrupados por estaciones. De hecho, los vientos sur y sureste son los más frecuentes en verano, situación que se asemeja a las estaciones de otoño y primavera, pero con vientos del SE y SW (Fig. 6). El invierno es diferente, pues la componente oeste es la más importante en Chile central.

Siguiendo con el análisis de tendencias estacional, esta vez para los tipos de circulación más típicos, encontramos nuevamente que el tipo A muestra disminución en todas las estaciones del año (Tabla 9). Anteriormente (Tabla 2), se señaló que los advectivos de circulación anticiclónica aumentaban, pero de manera no significativa. No obstante, los tipos que aumentan con p-valor $<0,05$ son los días AS (verano) y ASW (verano, otoño y primavera), y los que disminuyen en el subperíodo 1981-2010 son los días ASE, tanto en verano y otoño como en primavera. Los días ciclónicos (C) tienen un aumento significativo sólo en otoño, y los ciclónicos del sureste (CSE) disminuyen en primavera. Finalmente, el tipo advectivo del sur (S) se ha incrementado en los últimos 30 años (a excepción de invierno), y el del suroeste (SW) hace lo propio en otoño y primavera, mientras que el advectivo del sureste (SE) decrece en verano y otoño.

Adicionalmente se han observado las anomalías del índice del Oeste (WI). Dicho índice posee un régimen invernal, con máximos entre mayo y agosto, siendo verano cuando hay menos días con esta circulación (Tabla 9). Al aplicar análisis espectral la frecuencia del WI es de 0,083 , lo cual revela un máximo cada 12 meses. Un segundo máximo en el espectro de potencia se observa a una frecuencia de 0,166 (6 meses), lo cual evidencia el régimen invernal del WI.

Tabla 9

ÍNDICE DEL OESTE A NIVEL MENSUAL, NÚMERO MEDIO Y DESVIACIÓN ESTÁNDAR DE DÍAS CON CIRCULACIÓN DEL OESTE EN CHILE CENTRAL, ENTRE 1950 Y 2010

\begin{tabular}{|l|r|r|r|}
\hline \multicolumn{1}{|c|}{ Meses } & \multicolumn{1}{|c|}{$\begin{array}{c}\text { Índice del Oeste (WI) } \\
(\%)\end{array}$} & $\begin{array}{c}\text { No de días promedio con } \\
\text { circulación del Oeste }\end{array}$ & $\begin{array}{c}\text { Desviación estándar } \\
\text { (días) }\end{array}$ \\
\hline Enero & $13,3 \%$ & 4 & 3 \\
\hline Febrero & $12,4 \%$ & 4 & 4 \\
\hline Marzo & $19,2 \%$ & 9 & 4 \\
\hline Abril & $30,7 \%$ & 16 & 5 \\
\hline Mayo & $52,2 \%$ & 17 & 5 \\
\hline Junio & $57,9 \%$ & 18 & 3 \\
\hline Julio & $57,9 \%$ & 15 & 4 \\
\hline Agosto & $48,8 \%$ & 12 & 4 \\
\hline Septiembre & $39,7 \%$ & 9 & 3 \\
\hline Octubre & $28,2 \%$ & 6 & 4 \\
\hline Noviembre & $19,9 \%$ & 5 & 4 \\
\hline Diciembre & $15,6 \%$ & & \\
\hline
\end{tabular}


Anomalías del Western Index en Chile central

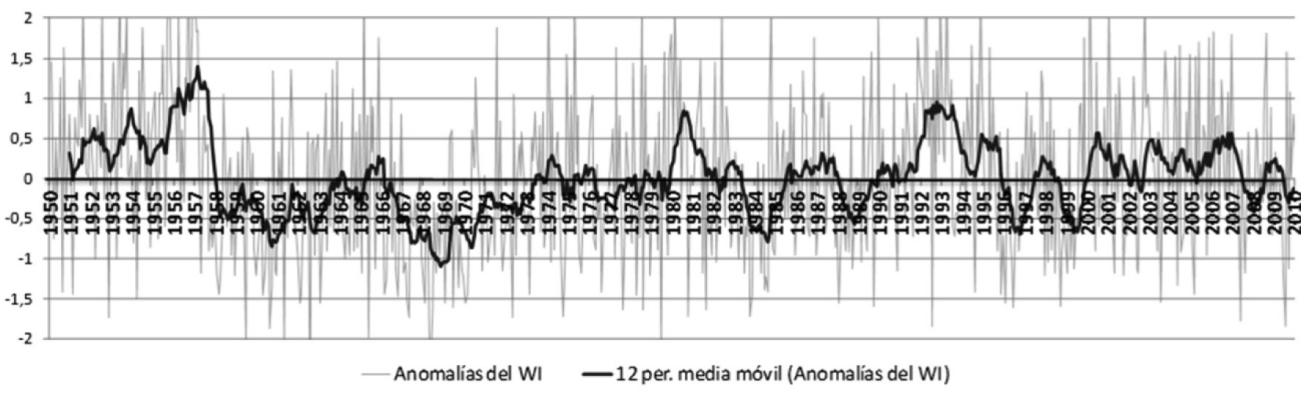

No obstante, las anomalías del WI muestran evidentes ciclos del índice, que revelan, por ejemplo, que entre 1950 y 1958 la tendencia fue de anomalía positiva, mientras que en el período de 1959 a 1980 la tendencia es negativa (Fig. 7). Esto, desde el punto de vista de la circulación atmosférica, es muy relevante, pues, aplicando un análisis espectral, se ha detectado que dichas anomalías poseen un ciclo de 146,4 meses (frecuencia de 0,007), lo cual corresponde a 12,2 años. Por lo tanto, habría que indagar si esto se correlaciona con patrones de baja frecuencia, sea El Niño Oscilación del Sur, la Oscilación Decadal del Pacífico o con los ciclos solares.

\section{Relación entre los tipos de J\&C y las teleconexiones SOI, MEI y PDO}

Se han correlacionado los 9 tipos de circulación más típicos de J\&C con las teleconexiones que influyen en Chile central, vale decir, la Oscilación Antártica, El Niño Oscilación del Sur (ENSO, medido con los índice SOI y MEI) y la Oscilación Decadal del Pacífico.

Los resultados indican que para el tipo A de la clasificación de Jenkinson Collison existe una relación significativa (p-valor de 0,000) e inversa con la Oscilación Antártica (Tabla 10), lo que sugiere que durante una fase negativa de la AAO Chile central presenta más días anticiclónicos, lo cual es consistente con las precipitaciones y las borrascas más meridionales. El estadístico $\mathrm{R}^{2}$ indica que el modelo ajustado explica el 36,48\% de la variabilidad del tipo A. El coeficiente de correlación es igual a -0,60, es decir, una relación moderadamente fuerte entre las variables. El error estándar del modelo indica que la desviación estándar de los residuos es de 22,32 días/año. El resto de los índices (SOI, MEI y PDO) no poseen relaciones estadísticamente significativas con los días anticiclónicos.

Los días híbridos de circulación anticiclónica del sur (AS) no se relacionan de manera significativa con ninguno de los patrones de teleconexiones, pero sí lo hacen con la Oscilación Antártica los tipos anticiclónicos del sureste (ASE, relación inversa) y del suroeste (ASW, relación directa).

El tipo ciclónico (Tabla 10) también se correlaciona significativamente con la Oscilación Antártica, con un p-valor de 0,009, y existe una relación directa entre el desarrollo de la actividad ciclónica en Chile central y la fase positiva de la AAO, lo cual resulta llamativo, pues 
en esta fase el frente polar se retrae, lo cual implica que la actividad ciclónica de Chile central tiene otro origen durante la fase positiva de la AAO. El $\mathrm{R}^{2}$ indica que el modelo explica el $11,01 \%$ de la variabilidad de los días ciclónicos. El coeficiente de correlación es de 0,33, lo que muestra una relación relativamente débil entre las variables. El error estándar indica que la desviación estándar de los residuos es 9,2 días.

Los tipos advectivos S y SW también se correlacionan con la Oscilación Antártica, ambas con signo positivo y con un $\mathrm{R}^{2}$ de $21,67 \%$. El tipo $\mathrm{S}$, además, se correlaciona con el SOI, de modo positivo. Ello puede indicar que, frente a fases positivas del índice de Oscilación del Sur (La Niña), hay mayor frecuencia de circulaciones advectivas del Sur, asociadas tradicionalmente a «buen tiempo».

Tabla 10

CORRELACIONES ENTRE LOS TIPOS SINÓPTICOS DE J\&C MÁS FRECUENTES DE CHILE CENTRAL ENTRE 1950-2010 Y LOS PATRONES DE TELECONEXIONES AAO, MEI, SOI Y PDO

\begin{tabular}{|c|c|c|c|c|}
\hline Tipos sinópticos & Regresión lineal con AAO & $\begin{array}{c}\text { Regresión } \\
\text { lineal con } \\
\text { MEI }\end{array}$ & $\begin{array}{l}\text { Regresión lineal } \\
\quad \text { con SOI }\end{array}$ & $\begin{array}{c}\text { Regresión } \\
\text { lineal con } \\
\text { PDO }\end{array}$ \\
\hline $\begin{array}{l}\text { Anticiclónico } \\
\text { (A) }\end{array}$ & $\begin{array}{c}\mathrm{A}=-22,15 \mathrm{AAO}+107,44 \\
\left(\mathrm{R}^{2}=36,48 \%\right)\end{array}$ & No sig. & No sig. & No sig. \\
\hline $\begin{array}{l}\text { Anticiclónico del } \\
\text { sur (AS) }\end{array}$ & No sig. & No sig. & No sig. & No sig. \\
\hline $\begin{array}{l}\text { Anticiclónico del } \\
\text { suroeste (ASW) }\end{array}$ & $\begin{array}{c}\mathrm{ASW}=2,815 \mathrm{AAO}+15,109 \\
\left(\mathrm{R}^{2}=19,12 \%\right)\end{array}$ & No sig. & No sig. & No sig. \\
\hline $\begin{array}{l}\text { Anticiclónico del } \\
\text { sureste (ASE) }\end{array}$ & $\begin{aligned} \mathrm{ASE}= & -2,203 \mathrm{AAO}+11,371 \\
& \left(\mathrm{R}^{2}=10,21 \%\right)\end{aligned}$ & No sig. & No sig. & No sig. \\
\hline Ciclónico (C) & $\begin{array}{c}\mathrm{C}=4,23 \mathrm{AAO}+31,91 \\
\left(\mathrm{R}^{2}=11,07 \%\right)\end{array}$ & No sig. & No sig. & No sig. \\
\hline $\begin{array}{l}\text { Ciclónico del } \\
\text { sureste (CSE) }\end{array}$ & No sig. & No sig. & No sig. & No sig. \\
\hline $\begin{array}{l}\text { Advectivo del } \\
\text { suroeste (SW) }\end{array}$ & $\begin{array}{c}\mathrm{SW}=1,452 \mathrm{AAO}+9,586 \\
\left(\mathrm{R}^{2}=6,41 \%\right)\end{array}$ & No sig. & No sig. & No sig. \\
\hline $\begin{array}{l}\text { Advectivo del } \\
\text { sureste (SE) }\end{array}$ & No sig. & No sig. & No sig. & No sig. \\
\hline $\begin{array}{l}\text { Advectivo del } \\
\quad \operatorname{sur}(\mathrm{S})\end{array}$ & $\begin{array}{c}\mathrm{S}=8,323 \mathrm{AAO}+46,036 \\
\left(\mathrm{R}^{2}=21,67 \%\right)\end{array}$ & No sig. & $\begin{array}{c}\mathrm{S}= \\
5,068 \mathrm{SOI}+42,323 \\
\left(\mathrm{R}^{2}=6,54 \%\right)\end{array}$ & No sig. \\
\hline
\end{tabular}

No sig. Significa que no hay relación estadísticamente significativa entre los tipos sinópticos y los índices de teleconexiones.

\section{CONCLUSIONES}

Predominan en la serie completa de 61 años (1950-2010) los días anticiclónicos, con una mayor frecuencia de los mismos en los meses invernales, cuando el APSO se sitúa en 
una posición más centrada con respecto a la malla usada en el análisis, e induce una mayor vorticidad negativa a los tipos sinópticos que se dan diariamente. En este sentido, los días de vorticidad anticiclónica (ANT) alcanzan un porcentaje del 54\% respecto del total, y esto se debe a la persistencia del APSO en estas latitudes. Sin embargo, el análisis de tendencia muestra una disminución de los tipos A en todas las estaciones, mientras que los días ADVA aumentan de manera significativa.

Los tipos híbridos anticiclónicos del sur (AS) son un caso particular, y es que, a parte de ser más frecuentes en el verano austral, no presentan relación significativa con ninguna de las teleconexiones estudiadas. Sin embargo, los tipos híbridos anticiclónicos del sureste (ASE) y del suroeste (ASW) presentan una relación significativa con la AAO, de manera inversa en el primer caso y de manera directa en el segundo. Esta relación se muestra acorde con la mayor frecuencia de los ASE en verano, cuando la AAO es menos persistente, y la mayor frecuencia de los ASW en invierno, cuando la AAO se acentúa.

El tipo ciclónico (C) es más frecuente entre marzo y octubre, en los meses invernales, gracias al avance del CBPP a costa del APSO, que ejercía bloqueo durante el verano. Este tipo se relaciona directamente con la AAO, coincidente con su fase positiva, cuando el frente polar se retrae, por lo que habría que buscar otro origen a esta actividad ciclónica.

Las circulaciones del sur y del sureste son las más frecuentes en verano, de noviembre a marzo, mientras que aquellas circulaciones de componente oeste se dan más entre mayo y octubre. De estas, la primera presenta una relación directa y significativa tanto con la AAO como con el SOI, mismo caso que con el tipo advectivo del suroeste con la AAO.

Considerando el WI para Chile Central, son más frecuentes los días de componente oeste entre mayo y octubre, como bien indica el análisis espectral, dando un máximo de invierno. Sin embargo, considerando los valores mensuales de toda la serie, aparece una periodicidad de 12,2 años, con una relación aún por determinar.

Conviene señalar que, de los 22.280 días estudiados, no se da ningún caso catalogado como indeterminado (U), y ello se debe a la inexistencia de situaciones de pantano barométrico, es decir, con escaso gradiente de presión atmosférica.

Por último, el catálogo diario de los tipos de $\mathrm{J} \& \mathrm{C}$ los ofrecemos en el portal web del Grupo de Climatología de la Universidad de Barcelona para que otros investigadores utilicen esta valiosa información, accediendo a http://www.ub.edu/gc/English/JC_Chile.htm, información que esperamos mantener actualizada.

\section{AGRADECIMIENTOS}

Los autores agradecen la cesión de datos del proyecto NCEP/NCAR Reanalysis, adquiridos a través de su sitio Web http://www.esrl.noaa.gov/psd/, sin los cuales este trabajo no hubiera sido posible. Además, esta investigación ha sido en parte realizada en el marco de desarrollo de una tesis doctoral financiada por el Programa de Formación de Profesorado Universitario del Ministerio de Educación de España. Esta investigación se inserta en el proyecto PRECABAL (CGL2011-29263-C02-01) del Ministerio de Ciencia e Innovación de España y en el Grupo de Climatología SGR 443 (Generalitat de Catalunya). 


\section{BIBLIOGRAFÍA}

EL-DESSOUKY, T.M. y JENKINSON, A.F. (1975): «An objective daily catalogue of surface pressure, flow and vorticity indices for Egypt and it's use monthly rainfall forecasting». Synoptic Climatology Branch Memorandum n ${ }^{\circ} 46$, Bracknell, Meteorological Office, London.

FRIAS, T.F. (2008): Padrões de circulação atmosférica no Chile. Tese de Mestrado em Ciências Físicas (Meteorologia), Universidade de Lisboa, 111 pp. (Inédita).

FRIAS, T.F., TRIGO, R. y GARREAUD, R. (2009): «Weather type classification over Chile; patterns, trends, and impact in precipitation and temperature». Geophysical Research Abstracts, Vol. 11, EGU-8432.

GRIMALT, M., TOMÀS, M., ALOMAR, G., MARTIN-VIDE, J. y MORENO-GARCÍA, M.C. (2013): «Determination of the Jenkinson and Collison's weather types for the Western Mediterranean basin over the 1948-2009 period. Temporal analysis». Atmosfera, 26, 75-94.

HESS, P. y BREZOWSKY, H. (1952): «Katalog der grosswetterlagen Europas». Berichte Deutsche Wetterdienstes 15(113): 39.

JENKINSON, A.F. y COLLISON, P. (1977): An initial climatology of gales over the North Sea. Synoptic Climatology Branch Memorandum n ${ }^{\circ}$ 62, Bracknell, Meteorological Office, London.

JONES, P.D., HULME, M. y BRIFFA K.R. (1993): «A comparison of Lamb circulation types with an objective classification scheme». International Journal of Climatology, 13, 655-663.

JONES, P.D., HARPHAM, C. y BRIFFA K.R. (2013): «Lamb weather types derived from reanalysis products». International Journal of Climatology, 33, 1129-1139.

KALNAY, E., KANAMITSU, M., KISTLER, R., COLLINS, W., DEAVEN, D., GANDIN, L., IREDELL, M., SAHA, S., WHITE, G., WOLLEN, J., ZHU, Y., CHELLIAH, M., EBISUZAKI, W., HIGGINS, W., JANOWIAK, J., MO, KC., ROPELEWSKI, C., WANG, J., LEETMAA, A., REYNOLDS, R., JENNE, R. y JOSEPH, D. (1996): «The NCEP/ NCAR 40 year reanalysis project». Bulletin of the American Meteorological Society, 77, 437-471.

LAMB, H.H. (1972): «British Isles weather types and a register of daily sequence of circulation patterns, 1861-1971». Geophysical Memoir, 116, HMSO, London, 85. pp.

LINDERSON, M. (2001): «Objective classification of atmospheric circulation over southern Scandinavia». International Journal of Climatology, 21, 155-169.

LUND, IA. (1963): «Map-pattern classification by statistical methods». Journal of Applied Meteorology, 2, 56-65.

MARTÍN-VIDE J. (2002): «Aplicación de la clasificación sinóptica automática de Jenkinson y Collison a días de precipitación torrencial en el este de España». En: Cuadrat, J.M., Vicente, S.M., y Saz, M.A. (Eds.), La información climática como herramienta de gestión ambiental. Zaragoza, Universidad de Zaragoza. pp. 123-127.

MARTÍN-VIDE, J. (2005): Los mapas del tiempo, Mataró, Davinci Continental, 221 pp.

POST, P., TRUIJA, V. y TUULIK, J. (2002): «Circulation weather types and their influence on temperature and precipitation in Estonia». Boreal Environment Research, 7, 281-289. 
QUINTANA, J. y ACEITUNO, P (2006): «Trends and interdecadal variability of rainfall in Chile». Proceedings of 8 ICSHMO, Foz do Iguaçu, Brazil, April 24-28, 2006, INPE, pp. 371-372.

QUINTANA, J. y ACEITUNO, P. (2012): «Changes in the rainfall regime along the extratropical west coast of South America (Chile): $30-43^{\circ}$ S». Atmósfera, 25(1), 1-22.

ROMERO, H. (1985): Geografía de los climas de Chile. Tomo XI Colección de Geografía de Chile. Instituto Geográfico Militar (IGM). 237 pp.

SARRICOLEA, P., MESEGUER-RUIZ, O. y MARTÍN-VIDE, J. (2011): «Aplicación de la clasificación automática de Jenkinson y Collison a Chile Central». Conferencia Geográfica Regional de la Unión Geográfica Internacional (UGI), (p. 10).Santiago.

SPELLMAN, G. (2000): «The use of an index-based regression model for precipitation analysis on the Iberian peninsula». Theoretical and Applied Climatology, 66, 229-239.

ŠTĚPÁNEK, P. (2003): AnClim - software for time series analysis. Dept. of Geography, Fac. of Natural Sciences, MU, Brno. 1.47 MB.

STEHLÍK, J. (2001): «Weather to weather links: relationships between Czech circulation pattern classification and other European regional classification schemes». Acta Universitatis Carolinae. Geographica, 2, 155-167.

TANG, L., CHEN, D., KARLSSON, P., GU, y OU, T. (2009): «Synoptic circulation and its influence on spring and summer surface ozone concentration in southern Sweden». Boreal Environment Research, 14, 889-902.

TRIGO, R. y DACAMARA, C. (2000): «Circulation weather types and their influence on the precipitation regime in Portugal». International Journal of Climatology, 20, 1559-1581.

WHEELER, D., GARCIA-HERRERA, R., WILKINSON, C.W. y WARD, C. (2010): «Atmospheric circulation and storminess derived from Royal Navy logbooks: 1685 to 1750». Climatic Change, 101, 257-280.

YARNAL, B., COMRIE, A.C., FRAKES, B. y BROWN, D. (2001): «Developments and prospects in synoptic climatology». International Journal of Climatology, 21, 1923-1950

ZHANG, Y., WALLACE, J.M. y BATTISTI, D.S (1997): «ENSO-like interdecadal variability: 1900-93». Journal of Climate, 10, 1004-1020. 
